\title{
Hydrothorax due to extravasation of intravenous contrast through left subclavian catheter -A case report-
}

\author{
Jung-Gi Choi ${ }^{1}$, June-Seog Choi ${ }^{1}$, Cheon-Hee Park ${ }^{1}$, Cheol-Seung Lee ${ }^{1}$, Lee-Kyoung Kim ${ }^{1}$, and \\ Young-Ryool Chung ${ }^{2}$
}

Departments of ${ }^{1}$ Anesthesiology and Pain Medicine, ${ }^{2}$ Orthopedic Surgery, Gwangju Christian Hospital, Gwangju, Korea

Central venous catheterization is associated with a large number of complications, such as pneumothorax, hydrothorax, hemothorax, phlebothrombosis, pericardial tamponade, air embolism, aberrant placement and line sepsis. There are many case reports of the extravasation of various central venous catheter fluids, including the intravenous fluids, total parenteral nutrition and chemotherapeutic agents into the pleural cavity and mediastinum. These have led to hydrothorax, hydromediastinum and pericardial effusions. We report a case of the extravasation of intravenous contrast into the pleural cavity after dynamic CT through a left subclavian catheter. (Korean J Anesthesiol 2010; 58: 550-554)

Key Words: Central venous catheterization, Extravasation, Hydrothorax, Intravenous contrast.

A central venous catheter is useful for monitoring the cardiovascular status in the management of patients under anesthesia or with severe diseases as well as for providing intravenous routes in case it is difficult to secure peripheral venous catheters and supply routes for fluids [1]. However, an injection of contrast medium through a central venous catheter using a mechanical pump causes more pressure that can result in leakage. Due to such pressure, the central venous catheter might move out of the blood vessels and the contrast medium may spout into the adjoining structures, such as a pleural space or mediastinum. This can cause hypoxia, sepsis, and even cardiovascular collapse and dyspnea. Therefore, it is important to detect the symptoms as early as possible.

We report a case of the extravasation of intravenous contrast into the pleural cavity after dynamic CT through the left subclavian catheter.

\section{Case Report}

A 51-year-old female patient, $52 \mathrm{~kg}$ in weight and $155 \mathrm{~cm}$ in height, visited to receive total hip arthroplasty due to osteoarthritis caused by acetabular dysplasia in her left hip joint. There were no special findings in her personal medical history, including a recent physical examination, chest X-ray, electrocardiogram and blood test before surgery.

Before the operation, $20 \mathrm{mg}$ of famotidine and $0.2 \mathrm{mg}$ of glycopyrrolate were administered intramuscularly. Due to a spinal block, $10 \mathrm{mg}$ of $0.5 \%$ bupivacaine was injected into the

Received: September 22, 2009. Revised: 1st, October 6, 2009; 2nd, October 30, 2009. Accepted: November 2, 2009.

Corresponding author: June-Seog Choi, M.D., Department of Anesthesiology and Pain Medicine, Gwangju Christian Hospital, 264, Yangnimdong, Nam-gu, Gwangju 503-715, Korea. Tel: 82-62-650-5150, Fax: 82-62-671-7447, E-mail: juneston@shinbiro.com

(c) This is an open-access article distributed under the terms of the Creative Commons Attribution Non-Commercial License (http:// creativecommons.org/licenses/by-nc/3.0/), which permits unrestricted non-commercial use, distribution, and reproduction in any medium, provided the original work is properly cited. 
subarachnoid space and a lack of sensation in the 9th thoracic vertebrae was confirmed. For sedation during surgery, 1.0 mg of midazolam was injected and $5 \mathrm{~L} / \mathrm{min}$ of oxygen was administered through a face mask. The patient was placed under anesthesia for approximately two hours. The amount of blood loss, fluid administered during surgery and urine output was $400 \mathrm{ml}, 2,100 \mathrm{ml}$ and $950 \mathrm{ml}$, respectively. Arterial blood gas analysis immediately before completion of the surgery revealed the following: $\mathrm{pH}$ 7.386, $\mathrm{PaCO}_{2} 42.5 \mathrm{mmHg}, \mathrm{PaO}_{2} 164.7 \mathrm{mmHg}$, $\mathrm{HCO}_{3} 24.6 \mathrm{mM} / \mathrm{L}, \mathrm{SaO}_{2} 99.8 \%$, and $\mathrm{Hb} 10.1 \mathrm{~g} / \mathrm{dl}$.

When the patient arrived in the recovery room, her blood pressure, heart rate and pulse oximetry was 110/60 $\mathrm{mmHg}$, 65 beats/min, and 99\%, respectively. The patient was covered with a warm blanket because she complained of being cold and shivered. After approximately three hours, a blood test, a chemical test and coagulation were performed because she complained of dizziness and showed slight cyanosis in her lips. At this time, her blood pressure, heart rate and pulse oximetry was $90 / 50 \mathrm{mmHg}, 70-95$ beats/min and $98 \%$, respectively. The test results showed $7.9 \mathrm{~g} / \mathrm{dl}$ of hemoglobin and $24.3 \%$ of hematocrit, due to suspected hypovolemia.

A decision was made to provide a central venous catheter to secure the routes for fluids and monitor the cardiovascular status. In addition an attempt was made to insert 7 Fr (Twolumen central venous subclavian catheter set with blue flex tip, Arrow, USA) to the left, because a large assortment of wires for monitors, fluids and racks were dangling and attached to the patient. The bed was inclined slightly to hold the patient in the Trendelenburg position. Her left arm was in complete internal rotation and her neck was turned to the right. Lidocaine was administered at 2-3 cm downward from themidpoint of the left central clavicle for local anesthesia and the skin was punctured using an $18 \mathrm{G}, 6.35 \mathrm{~cm}$ needle. The needle was inserted along the back of the clavicle to proceed toward the sternal notch parallel to the clavicle. A J-inducing wire was inserted after venipuncture and the needle was removed. Space for the catheter was expanded using an expander and after inserting the central venous catheter, it was confirmed that blood had been absorbed at the two catheters without resistance. They were fixed at an approximately $15 \mathrm{~cm}$ length from the skin. At this time, the patient's blood pressure, heart rate and pulse oximetry was $110 / 60 \mathrm{mmHg}, 78-100$ beats/min and 99\%, respectively. $500 \mathrm{ml}$ of packed red blood cells was injected through a central venous catheter.

The emergency blood test found that approximately $50 \mathrm{ml}$ of blood had drained into the Hemovac, which was much lower than that drained extracoporeally. Therefore, a decision was made to use computed tomography (CT) on the next morning to locate the bleeding, considering that $50 \mathrm{ml}$ of blood had drained into the Hemovac, which was too little. Iopromide, a contrast medium, was injected at a rate of $2 \mathrm{ml} / \mathrm{s}$ to a total dose of $120 \mathrm{ml}$ through a central venous catheter of the left subclavian vein for $\mathrm{CT}$ of the abdomen and pelvis.

Immediately after CT, the patient complained of heaviness in her chest and $5 \mathrm{~L} / \mathrm{min}$ of oxygen was administered through a face mask. At that time, her blood pressure and heart rate was 110/66 mmHg and 70 beats/min, respectively. The results of her arterial blood gas analysis were as follows: $\mathrm{pH}$ 7.406, $\mathrm{PaCO}_{2}$ $36.8 \mathrm{mmHg}, \mathrm{PaO}_{2} 109.6 \mathrm{mmHg}, \mathrm{HCO}_{3}{ }^{-} 11.5 \mathrm{mM} / \mathrm{L}$ and $\mathrm{SaO}$ 299.3\%. There were no outstanding symptoms evident after the electrocardiogram, an echo cardiogram and a cardiac enzyme test, and no signs of an allergic reaction that could occur after using the contrast medium. However, after a chest X-ray, the tip of the central venous catheter was placed at the brachiocephalic vein (Fig. 1) and a hydrothorax on the left was observed by abdominal CT.

At the department of radiology, the site of a hydrothorax was detected using a sono guide and a Chiba needle was inserted between the 8th and 9th rib to drain approximately $100 \mathrm{ml}$ of pleural effusion. An 8.5 F pig tail catheter was inserted using a hair wire. A slightly transparent fluid was absorbed through the piercing needle, which was checked by X-ray and established to be contrast medium. A contrast medium was injected slowly through the catheter to eliminate the possibility that it originated from the central venous catheter, which is a possible reason for it permeating through the chest cavity. It was confirmed that the contrast medium flowed inside the vein through the catheter and blood was sucked in slowly into the catheter without resistance. Although the central venous catheter was needed, a hair wire carefully was inserted to recheck the site of the catheter within the vein while double

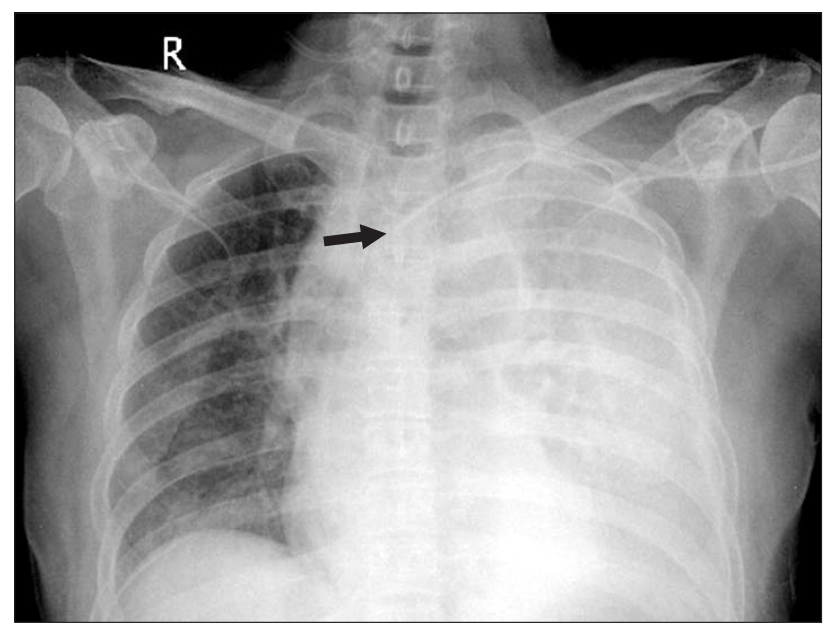

Fig. 1. Chest AP radiograph shows the pleural effusion of the left lung. The arrow shows that central venous catheter tip is positioned in the left brachiocephalic vein. 


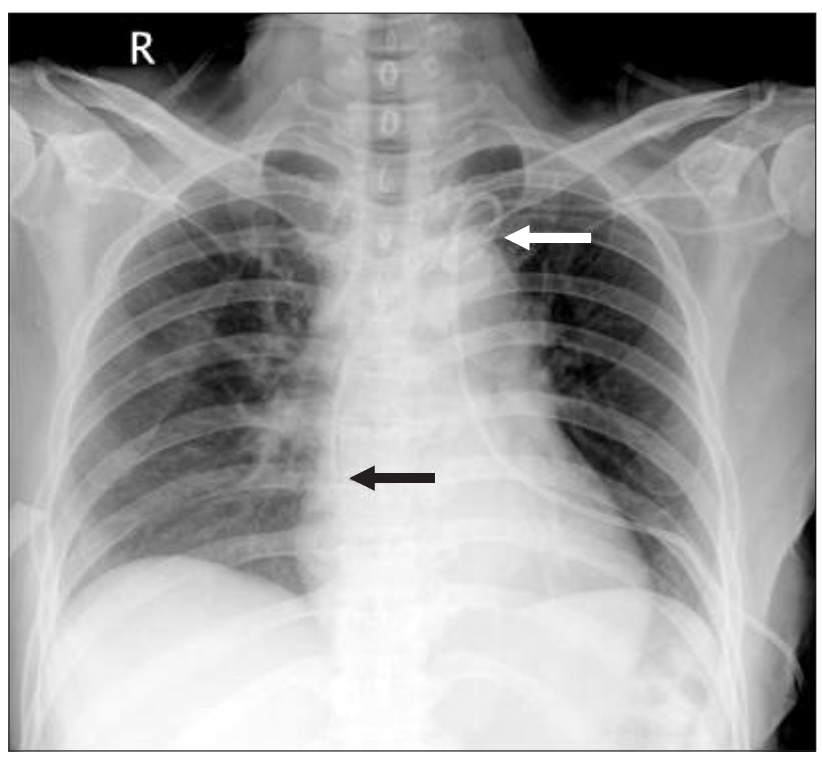

Fig. 2. Chest AP A radiograph shows the central venous catheter tip is positioned in the SVC and the pleural effusion disappears after thoracentesis (Black arrow: central venous catheter. White arrow: pig tail).

checking it with a sono guide and chest X-ray. It was replaced with another catheter and fixed $20 \mathrm{~cm}$ of its length away from the skin. Again we checked to see if blood was sucked into the catheter without resistance as well as its place using chest X-ray (Fig. 2).

The patient did not complain of dyspnea or any other symptoms and approximately $20 \mathrm{ml}$ of extracoporeal drainage was obtained with a pig tail catheter inserted in her chest cavity. The patient was observed after the surgery using a chest X-ray and her vital signs were monitored carefully to guard against anaphylaxis by the contrast medium or cardiopulmonary sideeffects.

Observations of her condition by chest X-ray showed that the hydrothorax had disappeared with no side-effects or cardiopulmonary symptoms. After seven days, the catheter was removed and the patient was moved to the general ward. Finally, she was discharged without a permanent lesion after 14 days.

\section{Discussion}

Given the current methods of medical care, central venous catheterization is useful for monitoring the cardiovascular state of a patient and administering drugs and fluids for the medical care of emergency patients, patients undergoing standard operations and patients undergoing critical care. However, approximately $15 \%$ of patients who undergo these procedures show a variety of complications $[1,2]$. Complications include pneumothorax, embolism, artery injury, septicemia, chest rupture, and phlebothrombosis. Due to a malposition of the central venous catheter, perforation of the vein can occur or fluids, chemotherapy or total parenteral nutrition leak to the adjoining structures, such as the chest cavity or mediastinum, which can cause hemothorax, hydromediastinum, hydrothorax, or cardiac tamponade. Such side-effects can cause fatal complications,such as chest pain, septicemia, due to hypoxia, even total cardiovascular collapse [2-4].

The position of the catheter tip must always be confirmed radiographically. Catheter tips located within the heart or below the pericardial reflection on the superior vena cava can increase the risk of cardiac perforation and fatal cardiac tamponade. Ideally, the catheter tip should lie within the superior vena cava, parallel to the vessel walls, and be positioned below the inferior border of the clavicles and above the level of the third rib T4 to T5 interspace, azygos vein, tracheal carina, or takeoff of the right main stem bronchus. The state of the catheter in the vein should be checked further and its placement in the vein be confirmed by measuring the venous pressure using a monitor [5]. Ruesch et al. [6] reported that even when experienced surgeons insert central venous catheters, approximately $14 \%$ of cases malposition can occur, and the frequency of hydrothorax due to the insertion of a subclavian central venous catheter is approximately $0.1-2 \%$ of cases [7].

Anesthesiologists prefer the right internal jugular vein for a central venous catheter as it is consistent in anatomical structures and predictable, and the doctors have easy access to the head of the patient during surgery. However, the subclavian vein is selected when the plasma volume is increased or treatment through the vein is required.

Furthermore, the experience and skill of the surgeons are important in deciding whether it is safer to pierce a particular part of the central venous catheter. The central venous catheter should be inserted deeper, approximately $3-5 \mathrm{~cm}$, when surgery is performed on the left side [5].

In this case, the subclavian vein was selected for the central venous catheter and the end of the catheter was positioned within the brachiocephalic vein (Fig. 1). The hydrothorax and hydromediastinum occurred when contrast medium was injected into the catheter because the fluid drained from the chest was found to be contrast medium that did not contain blood. There are a few causes of hydrothorax due to contrast medium.

First, a perforation occurred when the subclavian vein was pierced and the catheter was installed, and so the catheter might have been positioned outside the blood vessel. However, we checked blood was aspired into the catheter through which packed red blood cells were provided. The catheter was not positioned outside the vessel because there was no blood found 
in the thoracentesis.

Second, after the catheter was positioned within the vessel, its end might have project from the pleural cavity around the mediastinum out of the vessel due to an erosion of the vessel walls or vascular rupture caused by a change in position. With such causes, the distal end of the catheter touched to the wall of the vessel, and after a certain period of time, ongoing stimulation to the end occurred due to respiratory movement, cardiac wall movement, and a change in the patient's position, which caused erosive damage. The end of the catheter might have been pulled out of the vessel and perforated it $[8,9]$. Such cases are called late-onset puncture, and hydromediastinum or hydrothorax can occur due to a puncture caused by longterm fluid injection. With respect to this case, there are no examples of the catheter being positioned outside the vessel, nor by contrast medium through the catheter $[3,4]$. However, in the present case, the contrast medium was slowly injected into the catheter to confirm its proper positioning and there is no possibility of incorrect positioning of the end due to erosive abrasion.

Third, the high infusion pressure of the contrast medium pump at the end of the catheter inside the vessel may cause vessel puncture. In this case, it was administered at a rate of $2 \mathrm{ml} / \mathrm{s}$. In particular, it was reported that because of the high viscosity of the contrast medium, the pressure from the catheter was higher than that of thenormal fluid injected at same infusion rate [10]. Lee et al. [4] reported cases of death due to cardiac tamponade because a mediastinum hematoma occurred just four hours after a blood pump had been used for the catheter, which indicates that vessels or heart walls may be perforated due to the high infusion pressure. In this case, it is possible that blood walls may be perforated and a hydrothorax occurs because of the high pressure at the position where the end of the catheter touches the walls of a blood vessel when a contrast medium is injected into the catheter using a pump. However, in the case of perforation due to pressure, there is a high likelihood that the blood inside the vessel with a contrast medium flows rapidly into the chest. Therefore, the possibility of perforation within blood vessels was low considering that the hydrothorax drained contained only a contrast medium rather than blood.

Fourth, backward movement of the catheter due to the infusion pressure when the contrast medium is injected can result in it being positioned outside the vessel from a perforated subclavian vein to the catheter where the contrast medium was injected. The double rumen used by the authors has a total of three holes through which fluid flows. There was an oval shaped hole, $1 \mathrm{~mm}$ in diameter, which was $2.5 \mathrm{~cm}$ away from the end of the catheter at the $14 \mathrm{G}$ fluid line to the distal off the catheter as well as an oval-shaped hole with a diameter of $4 \mathrm{~mm}$ at the side of the catheter, which was $5 \mathrm{~cm}$ away from the end of the catheter on the $18 \mathrm{G}$ fluid line proximal to the catheter. Therefore, it was estimated that the proximal fluid hole inside the subclavian vein was adjacent to the perforation of the subclavian vein because the length to the catheter from the end of the hole at the proximal fluid line was $5.4 \mathrm{~cm}$, and the length of the catheter inserted was short. In this state, it was estimated that the catheter had been pushed outside the vessel due to the high infusion pressure of the pump and the contrast medium that was injected through the proximal fluid hole effused outside the vessel. The proximal fluid hole returned to its original place due to the elasticity of the catheter when the injection pressure disappeared.

In this state, it was estimated that the catheter had been pushed outside the vessel due to the high infusion pressure of the pump and the contrast medium that was injected through the proximal fluid hole effused outside the vessel. The proximal fluid hole returned to its original place due to the elasticity of the catheter when the injection pressure disappeared.

It is possible that hydrothorax will be not caused by the contrast agent if it is injected into the distal hole rather than the proximal hole, even though the catheter may be pushed by the high injection pressure and a fluid hole is exposed outside the vessel.

Colin et al. [11] reported that after CT of the chest through a catheter positioned to the left subclavian vein, a patient had a hydromediastinum caused by the effusion of contrast medium. However, the contrast medium at the mediastinum disappeared according to the chest X-rays taken after 24 hours. Nevertheless, the most suitable treatment is to aspire or drain out the components of the contrast medium effused outside a vessel to affect complete drainage because tissue necrosis can occur due to cytotoxicity according to components, concentration and volume of contrast medium [12]. Careful observation of the side effects, such as anaphylaxis and vital signs, is needed.

There is considerable controversy regarding how to inject a contrast medium through a catheter. Carlson et al. [13] reported that the use of a contrast medium pump through a catheter is safe. Ruess et al. [10] presented clinical data on pressure according to the sizes and types of catheters. The pressure caused by the rate of Iopromide used in this case $(2 \mathrm{~m} / \mathrm{s})$ was approximately $35 \mathrm{Psi}$, which is 50 Psi below that recommended by the manufacturer.

In conclusion, as a small part of the catheter was inserted through the subclavian vein and the proximal catheter hole for infusion of a contrast medium, it was exposed outside the vessel and contrast medium was effused to the chest cavity as a result of its infusion pressure. Therefore, fatal complications can occur when a central venous catheter is used for nutrition fluids, chemotherapy or a contrast medium for X-rays rather 
than general fluids due to the extravasation of intravenous medicine. Therefore careful attention should be paid to the proper positioning of the central venous catheter. Finally, a distal catheter is better for injecting medicine than a proximal one.

\section{References}

1. Taylor RW, Palagiri AV. Central venous catheterization. Crit Care Med 2007; 35: 1390-6.

2. McGee DC, Gould MK. Preventing complications of central venous catheterization. N Engl J Med 2003; 348: 1123-33.

3. Diaz M, Lorda I, Sanchez C, Gutierrez I, Monton JM, Vidal A. Milkywhite pleural effusion complicating peripherically inserted central venous catheter for total parenteral nutrition. Eur J Clin Nutr 2005; 59: 302-3.

4. Lee KH, Lee KM, Lee YB, Lim HK, Han JW. Hemomediastinum caused by central venous catheter. Korean J Anesthesiol 1998; 35: 1216-20.

5. Jonathan BM, Thomas FS. Miller's anesthesia. 6th ed. Philadelphia, Elsevier. 2005, pp 1287-97.

6. Ruesch S, Walder B, Tramer MR. Complication of central venous catheters: internal jugular versus subclavian access-a systemic review. Crit Care Med 2002; 30: 454-60.

7. James PM, Myers RT. Central venous pressure monitoring: complications and a new technique. Am Surg 1973; 39: 75-81.

8. Ellis LM, Vogel SB, Copeland EM 3rd. Central venous catheter vascular erosion. Diagnosis and clinical course. Ann Surg 1989; 209: 475-8.

9. Duntley P, Siever J, Korwes ML, Harpel K, Heffner JE. Vascular erosion by central venous catheters. Clinical features and outcome. Chest 1992; 101: 1633-8.

10. Ruess L, Bulas DI, Rivera O, Bruce M, Markle BM. In-line pressures generated in small-bore contral venous catheters during power injection of CT contrast media. Radiology 1997; 203: 625-9.

11. Colin S, Elijah D, Paul B, Chad GB, Andrew WK. Extravasation of intravenous contrast into the mediastinum. Inj Extra 2006; 37: 173-5.

12. Bellin MF, Jakobsen JA, Tomassin I, Thomsen HS, Morcos SK, Thomsen HS, et al. Contrast medium extravasation injury: guidelines for prevention and management. Eur Radiol 2002; 12: 2807-12.

13. Carlson JE, Hedlund LJ, Trenkner SW, Ritenour R, Halvorsen RA Jr. Safety considerations in the power injection of contrast media via central venous catheters durng computed tomographic examinations. Invest Radiol 1992; 27: 337-40. 